\title{
IVD.04 - Leishmania infantum lipophosphoglycan, a novel carbohydrate- based antigen for the immunodiagnosis of canine visceral leishmaniasis
}

Rodrigo Pedro Soares ${ }^{1 *}$; Ricardo Wagner Dias Portela²; Gabriela Porfírio-Passos ${ }^{2}$; Thiago Doria Barral²; Rafaela S. Gonçalves²; Marcos F. Bernardo²; Stella Maria Barrouin-Melo².

1 Fiocruz/CPqRR;

2UFBA - Universidade Federal da Bahia.

Introduction: Canine visceral leishmaniasis (CVL) is caused by Leishmania infantum, whose immunodiagnosis is crucial for disease control and treatment. Lipophosphoglycan (LPG) is the major Leishmania surface glycoconjugate. Current available diagnostic tests lack sensitivity especially for the diagnosis of asymptomatic dogs. Several diagnostic tests including EIE and DPP (Bio-Manguinhos) are based on protein antigens. Here, we propose a carbohydratebased antigen (LPG), for CVL immunodiagnosis.

Objective: Evaluating LPG as an antigen for CVL immunodiagnosis.

Methodology: We have used a standardized ELISA protocol using LPG as the antigen. Serum samples from dogs with a confirmed diagnostic of Leishmania infection and negative controls were used. For differential comparison, serum samples from dogs infected with Leishmania braziliensis, Trypanosoma cruzi and other pathogens were used. This work was approved by the CEUA from UFBA (023/2013). Validation parameters included specificity, sensitivity, negative and positive predictive values (NPV and PPV). ROC curve was obtained for each ELISA and the accuracy was defined as the area under the curve (AUC) of each one of the ROC curves. Kappa (K) indexes were also provided.

Results: LPG-ELISA was characterized by a $98.5 \%$ specificity and $91.5 \%$ sensitivity compared to TLA-ELISA (98.5\% and $85.0 \%$ ) and DPP (93.1\% and 90.6\%), respectively. Negative predictive value (NPV) was higher for LPG-ELISA (89.3\%) than for TLA-ELISA (84.8\%) and DPP (86.3\%). The accuracies of the test were based on ROC curve were higher for LPG-ELISA (99,7\%) than for TLA-ELISA (98.6\%). LPG-ELISA did not have any cross-reaction with $T$. cruzi and L. braziliensis. When positive samples were classified according to the clinical stage of the animal, it was possible to see that LPG-ELISA was able to identify $9 / 10$ (90\%) of the stage 01 asymptomatic and infected animals as positive, while just one sample (10\%) presented such result in TLA-ELISA and six (60\%) in DPP. Those data showed the better ability of LPG antigen not only in detecting asymptomatic dogs but also those with clinical symptons compared to TLA and DPP. Also, DPP test gave false positives being able to cross-react with T. cruzi and L. braziliensis. Surprisingly, LPG from L. infantum did not cross-react even with $L$. braziliensis reinforcing the specifity of the LPG antigen. Also, kappa values in agreement with parasitological tests were higher for ELISA-LPG (0.9) than DPP (0.85).

Conclusion: L. infantum-derived LPG presented high efficacy for the detection of CVL dogs in comparison to DPP. The developed assay showed no cross-reactions witle sera from animals infected with other pathogens, and was able to identify $90 \%$ of asymptomatic dogs. The specifity, sensibility and absence of cross-reaction was superior to DPP. This antigen could be considered as a promising tool for CVL immunodiagnosis.

Keywords: Immunodiagnosis; Canine Visceral Leishmaniasis; Lipophosphoglycan 